\title{
Data Models and Protocol Mapping for Reduced Communication Load in Substation Automation with High Sampling Rate Protection Applications
}

\author{
$1^{\text {st }}$ Fabian Hohn \\ Electric Power \& Energy Systems \\ KTH Royal Institute of Technology \\ Stockholm, Sweden \\ fhohn@kth.se
}

\author{
$2^{\text {nd }}$ Lars Nordström \\ Electric Power \& Energy Systems \\ KTH Royal Institute of Technology \\ Stockholm, Sweden \\ larsno@kth.se
}

\begin{abstract}
In digital substations measurements between instrument transformers and protection systems are exchanged via an Ethernet-based process-level network using the Sampled Value (SV) protocol. At the same time, new protection applications, based on time-domain or travelling-wave signal information, require very high sampling rates, which increases the communication load on the network significantly. The increase of the communication load can be reduced through the concept of Distributed Signal Processing Units (DSPU). This paper proposes data models and suitable protocol mappings for such DSPUs and analyses their impact on the process-level network. The results are compared with the SV approach specified in IEC 61869-9 for digital interfaces of instrument transformers. It is shown that the DSPU concept can reduce the required network bandwidth in the case of high sampling rate protection applications.

Index Terms-Communication networks, Distributed processing, Data models, Protocols, Substation protection, Systems architecture
\end{abstract}

\section{INTRODUCTION}

The digitalization in substations has led to an increased deployment of industrial Ethernet-based process-level networks in order to replace conventional copper cables between the Intelligent Electronic Devices (IED) and the instrument transformers [1] that are interfaced through Merging Units (MUs). These types of process-level networks need to fulfil very stringent requirements for Sampled Value (SV) based protection applications, such as a maximum transfer time of $3 \mathrm{~ms}$, a bumpless communication recovery delay and a time synchronisation accuracy of $1 \mu \mathrm{s}$ [2]. The performance of these networks has been analysed in terms of the time synchronisation accuracy in [3] and in terms of network delays in [4]. The second major impact of digital substations is the high degree of functional integration of contemporary IEDs, which trends towards a centralized substation protection and control architecture (CPC), as shown in [5]. At the same time, conventional phasor based protection functions are complemented by time-domain and travelling wave based protection functions, which require high sampling rates of the current and voltage signals up to $1 \mathrm{MHz}$ [6]. These high sampling rates impose particular challenges for digital substations utilising an Ethernet-based process-level network and having a high degree of functional integration. The drawbacks are twofold. Firstly, publishing the signals with high sampling rates increases the communication burden significantly. Secondly, all the computationally expensive digital signal processing (DSP) algorithms are executed on the centralized platform, which leads to high computational requirements. In order to mitigate the first challenge of the high communication burden, [7] suggests to implement the travelling wave feature extraction, such as polarity and magnitude of the wave, on the MU and then to publish the extracted information on the process bus. The network engineering guidelines of the IEC 61850 standard [8] suggests to increase the transmission speeds or to apply network partitioning or traffic control in the case of high data traffic process-level networks. Considering the high sampling rate requirements of the travelling-wave based protection a simple increase of the transmission speed is not sufficient. The IEEE standard about "Recommended Practice for Signal Treatment Applied to Smart Transducer" indicates that signals will be entirely processed at the point of measurement in the future [9]. This notion is picked up by [10] and is applied to the application of power system protection in substations. The paper [10] suggests to integrate the required DSP algorithms of the protection function in a dedicated device termed a distributed signal processing unit (DSPU), which publishes the results of the DSP algorithms on the process-level network instead of the raw samples. Thus the high communication burden as well as the increased computational demand of the centralized platform are able to be reduced.

\section{A. Scope of the Paper}

The data modelling approach in IEC 61850 considers the signal processing algorithms that are needed for the protection functions to be part of the protection logical nodes (LN), e.g. PTOC for overcurrent protection. Hence, there are no dedicated signal processing LNs for protection purposes. Thus this paper builds on the DSPU concept proposed in [10] and derives suitable data models for the different signal processing algorithms. In addition, the paper describes the 

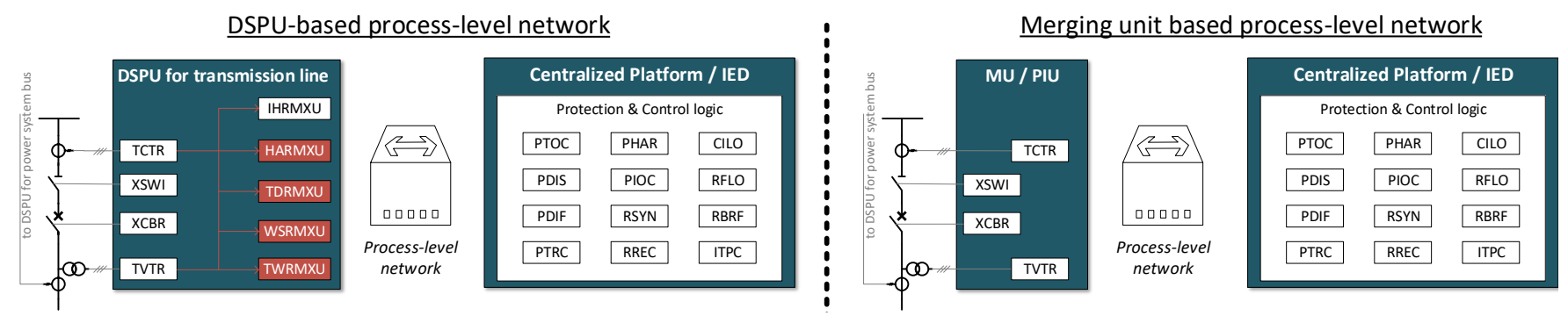

Fig. 1. Logical architecture of the DSPU-based and MU-based process-level substation network

mapping of the corresponding data sets to the Generic Object Oriented Substation Event (GOOSE) protocol. Furthermore, the impact of the derived data sets on the communication load of the process-level network is evaluated and compared with expected communication burden that is caused by the data sets of the IEC 61869-9 standard for digital interfaces of instrument transformers [11].

\section{B. Outline of the Paper}

The reminder of this paper is structured as follows. In Sec. II the DSPU-based process-level network is described, including how it differs in comparison to the MU-based process-level network. Then in Sec. III the data models of the DSPU are derived and explained. Furthermore, Sec. III discusses the considered IEC 61850 communication protocols. In Sec. IV the impact on the required network bandwidth is estimated for the DSPU and for the MU with respect to different substation protection applications. Lastly, Sec. V concludes the paper.

\section{DSPU-BASEd PROCESS-LEVEL Network}

The functional scope of the DSPU differs from the MU in terms of that, instead of directly publishing the sampled current and voltage signals on the process-level network, it processes the discrete-time signals by the necessary DSP algorithms first and then publishes the extracted signal information. This approach allows publishing the results of the DSP algorithms at a lower rate in comparison to the MU approach and thereby the publishing rate of the DSPU becomes independent of the sampling rate of its input signals. The logical architecture of these two concepts, in terms of their IEC 61850 Logical Nodes (LN) [12], is shown in Fig. 1 for an exemplary transmission line bay consisting of a 3-phase current transformer (CT) and voltage transformer (VT) as well as a circuit breaker and disconnector. It can be seen that the substation protection and control logic is located on the IEDs and on the centralized platform, respectively. Moreover, the MU comprises the LNs TCTR and TVTR, which correspond to the data models for the current and voltage transformers, respectively. These LNs provide the semantic model for the SV. Since the LNs $X C B R$ and $X S W I$ are included for the circuit breaker and disconnector, respectively, the term Process Interface Unit (PIU) is sometimes used in the literature [5]. On the other hand, the DSPU comprises five additional LN IHRMXU, HARMXU, TDRMXU, WSRMXU and TWRMXU, which refer to the DSP algorithms of the DSPU, namely interharmonic module, harmonic module, time-domain module, waveshape module and travelling wave module, respectively. These modules provide the required signal information to the protection logic, which have been modelled in Sec. III using the common data classes (CDC) defined in IEC 61850-73 [13]. Subsequently, the DSP results are published by the GOOSE application layer protocol, which is configured with a fixed and synchronised publishing rate, similar to [14] for wide-area networks (WAN). This paper focuses on the four LNs HARMXU, TDRMXU, WSRMXU and TWRMXU, marked in red in Fig. 1.

\section{DATA MOdELS FOR THE DSPU}

The data models in this paper are derived based on the modelling approach introduced by the standard IEC 61850. Thereby, new LNs are derived for the harmonic module, time-domain module, waveshape module and travelling-wave module of the DSPU, mentioned in Sec. II. Those new LNs are necessary, since the IEC 61850 standard considers the DSP algorithms for substation protection to be part of the respective protection $\mathrm{LNs}$ and does not allow to use the measurement LNs, e.g. MMXU, as an input to the protection LNs [2]. Hence, specific signal processing LNs do not exist for protection functions with the exemption of the LN RMXU, which can be used to model the phasor information exchange between local and remote line end for line differential protection. Throughout the data modelling process the standard data objects, defined in [12], have been used to a large extent. Nonetheless, new data objects had to be derived for the LNs $T D R M X U, W S R M X U$ and TWRMXU, which are all assigned to the CDC, defined in [13]. Lastly, suitable data sets are created for different transmission line protection applications and mapped to the GOOSE protocol, as described in [15].

\section{A. IEC 61850 Logical Nodes for DSPU}

The Tab. I defines the data objects of the derived LNs. It should be noted that only those data objects are shown that belong to the category of measured and metered values of the LNs HARMXU, TDRMXU and TWRMXU and that belong to the category of status information of the LN WSRMXU. Other important data objects need to be specified as well in the future. These are for example data objects for defining the 
applied calculation method or for specifying the method of assigning the timestamp to the data.

1) LN HARMXU: The harmonic module of the DSPU estimates most of the measurements needed for the conventional phasor based protection functions, such as the fundamental phasor information of the current and voltage signals as well as the $2^{\text {nd }}, 3^{\text {rd }}$ and $5^{\text {th }}$ harmonics of the current in order to detect inrush conditions or overexcitation situations of power transformers. Additionally, charging currents of long transmission lines are calculated by this module. Lastly, the harmonic module estimates the DC component of the current signal during transients, such as short-circuits. The phasor and the DC component information are modelled by the CDC complex measured value (CMV) and by the CDC measured value (MV), respectively. The CDC are shown in Tab. II.

2) $L N T D R M X U$ : The time-domain module estimates different quantities of the current and voltage signals, which are needed for directional based and distance based timedomain protection functions. These values are integrated measurements over time and can therefore be sent at a flexible publishing rate independent of the respective sampling rate. Since there are no predefined data objects for these type of quantities in [12], customized data objects have been derived. It should be noticed that all of the customized data objects of this LN are assigned to the CDC MV. In addition, the LN $T D R M X U$ comprises the data objects for the True-RMS values of the current signal.

3) LN WSRMXU: The waveshape analysis module of DSPU comprises the status information related to the sampled value based security and supervision functions, such as Mho impedance supervision, phase-to-phase current variation or current waveshape analysis, e.g. to detect inrush conditions of power transformers. This information is assigned to the CDC protection activation information (ACD), shown in Tab. II. Moreover, supervision information, related to open CT detection or fuse failure of VTs, is directly mapped to the quality attributes of the respective data object.

4) LN TWRMXU: The measured values of the travellingwave module are modelled by the data objects, which are required for the directional based and differential based travelling-wave protection. In case of the differential based travelling wave protection the magnitude and polarity of the first incident wave of the current signal are encoded in the data objects together with the timestamp of the first incident wave. The CDC MV has been used to model these customized data objects.

\section{B. IEC 61850 Data Sets}

Considering the data models of the previous section, the data sets are derived in order to group the data attributes that are required by different transmission line protection applications. These data sets provide the measured values for the phasor based, time-domain based and travelling wave based protection functions, as shown in Tab. III. The Tab. III depicts also the expected size of the data sets, which serve as a calculation basis in Sec. IV. For brevity the Tab. III
TABLE I

PROPOSED IEC 61850 LOGICAL NODES FOR DSP MODULES OF DSPU

\begin{tabular}{|c|c|c|}
\hline \multicolumn{3}{|c|}{ HARMXU LN } \\
\hline Data object & CDC & Explanation \\
\hline \multicolumn{3}{|c|}{ Measured and metered values } \\
\hline A.phsA & CMV & Current phasor A fundamental \\
\hline A.phsB & CMV & Current phasor B fundamental \\
\hline A.phsC & CMV & Current phasor $\mathrm{C}$ fundamental \\
\hline A.neut & CMV & Current phasor $\mathrm{N}$ fundamental \\
\hline PhV.phsA & CMV & Voltage phasor A fundamental \\
\hline PhV.phsB & CMV & Voltage phasor B fundamental \\
\hline PhV.phsC & CMV & Voltage phasor $\mathrm{C}$ fundamental \\
\hline PhV.neut & CMV & Voltage phasor $\mathrm{N}$ fundamental \\
\hline HA.phsAHar & CMV array & $2^{\text {nd }}, 3^{\text {rd }}, 5^{\text {th }}$ current harmonic phase $\mathrm{A}$ \\
\hline HA.phsBHar & CMV array & $2^{\text {nd }}, 3^{\text {rd }}, 5^{\text {th }}$ current harmonic phase $\mathrm{B}$ \\
\hline HA.phsCHar & CMV array & $2^{\text {nd }}, 3^{\text {rd }}, 5^{\text {th }}$ current harmonic phase $\mathrm{C}$ \\
\hline HA.neutHar & CMV array & $2^{\text {nd }}, 3^{\text {rd }}, 5^{\text {th }}$ current harmonic phase $\mathrm{N}$ \\
\hline A.phsA & CMV & Charging current of phase $\mathrm{A}$ \\
\hline A.phsB & CMV & Charging current of phase $B$ \\
\hline A.phsC & CMV & Charging current of phase $\mathrm{C}$ \\
\hline A.phsA & MV & DC component of current phase $\mathrm{A}$ \\
\hline A.phsB & MV & DC component of current phase B \\
\hline A.phsC & MV & DC component of current phase $\mathrm{C}$ \\
\hline \multicolumn{3}{|c|}{ TDRMXU LN } \\
\hline Data object & CDC & Explanation \\
\hline \multicolumn{3}{|c|}{ Measured and metered values } \\
\hline TdDirFwdA & MV & Forward directional quantity of phase A \\
\hline TdDirFwdB & MV & Forward directional quantity of phase B \\
\hline TdDirFwdC & MV & Forward directional quantity of phase $\mathrm{C}$ \\
\hline TdDirRevA & MV & Reverse directional quantity of phase $\mathrm{A}$ \\
\hline TdDirRevB & MV & Reverse directional quantity of phase B \\
\hline TdDirRevC & MV & Reverse directional quantity of phase $\mathrm{C}$ \\
\hline TdDirOpA & MV & Operate directional quantity of phase $\mathrm{A}$ \\
\hline TdDirOpB & MV & Operate directional quantity of phase $\mathrm{B}$ \\
\hline TdDirOpC & MV & Operate directional quantity of phase $\mathrm{C}$ \\
\hline TdDisValAG & MV & Distance quantity of fault-loop AG \\
\hline TdDisValBG & MV & Distance quantity of fault-loop BG \\
\hline TdDisValCG & MV & Distance quantity of fault-loop CG \\
\hline TdDisValAB & MV & Distance quantity of fault-loop $\mathrm{AB}$ \\
\hline TdDisValBC & MV & Distance quantity of fault-loop BC \\
\hline TdDisValCA & MV & Distance quantity of fault-loop CA \\
\hline A.phsA & MV & True-RMS value of current phase $\mathrm{A}$ \\
\hline A.phsB & MV & True-RMS value of current phase B \\
\hline A.phsC & MV & True-RMS value of current phase $\mathrm{C}$ \\
\hline \multicolumn{3}{|c|}{ WSRMXU LN } \\
\hline Data object & CDC & Explanation \\
\hline \multicolumn{3}{|c|}{ Status information } \\
\hline StrWav & ACD & Start of waveform restrain condition \\
\hline StrCurVar & ACD & Start of phase-to-phase current variation \\
\hline StrMhoSup & ACD & Start of Mho impedance supervision \\
\hline StrOpCT & $\mathrm{ACD}$ & Start of open CT detection \\
\hline StrAddSup & ACD & Start of other sampled-based supervision \\
\hline \multicolumn{3}{|c|}{ TWRMXU LN } \\
\hline Data object & CDC & Explanation \\
\hline \multicolumn{3}{|c|}{ Measured and metered values } \\
\hline TwDirValA & MV & Directional quantity of phase $\mathrm{A}$ \\
\hline TwDirValB & MV & Directional quantity of phase B \\
\hline TwDirValC & MV & Directional quantity of phase $\mathrm{C}$ \\
\hline TwDifValA & MV & Magnitude \& polarity of wave phase A \\
\hline TwDifValB & MV & Magnitude \& polarity of wave phase $\mathrm{B}$ \\
\hline TwDifValC & MV & Magnitude \& polarity of wave phase $\mathrm{C}$ \\
\hline
\end{tabular}


TABLE II

IEC 61850 COMMON DATA CLASSES USED FOR DATA MODELLING

\begin{tabular}{|l|l|l|l|}
\hline \multicolumn{5}{|c|}{ CDC complex measured value (CMV) } \\
\hline Data attribute & Type & Size & Explanation \\
\hline cVal.mag.i & int32 & 32 bit & Magnitude of complex value \\
\hline cVal.ang.i & int32 & 32 bit & Magnitude of complex value \\
\hline q & quality & 32 bit & Constructed quality type \\
\hline t & TimeStamp & 64 bit & UTC time stamp \\
\hline \hline \multicolumn{4}{|c|}{ CDC measured value (MV) } \\
\hline Data attribute & Type & Size & Explanation \\
\hline mag.i & int32 & 32 bit & Measured value \\
\hline q & quality. & 32 bit & Constructed quality type \\
\hline t & TimeStamp & 64 bit & UTC time stamp \\
\hline \hline \multicolumn{5}{|c|}{ CDC protection activation information (ACD) } \\
\hline Data attribute & Type & Size & Explanation \\
\hline phsA & boolean & 1 bit & Status indication for phase A \\
\hline phsB & boolean & 1 bit & Status indication for phase B \\
\hline phsC & boolean & 1 bit & Status indication for phase C \\
\hline q & quality. & 32 bit & Constructed quality type \\
\hline t & TimeStamp & 64 bit & UTC time stamp \\
\hline
\end{tabular}

depicts the data attributes corresponding to one of the phases only and therefore the size of the data attribute is multiplied by the number of phases or measurement loops, respectively, as indicated by the third column. Additionally, three different types of transmission lines have been considered, which differ in terms of the required measured values by the phasor based protection functions. Therefore the phasor based protection data set PhsProtLine consists of a set of measured values that are always required by the transmission line protection logic, and of two additional sets of measured values that are needed for long transmission lines and transmission lines with an inzone transformer, respectively.

\section{Communication Protocol Mapping}

The different data sets, described in Sec. III-B, are mapped to the GOOSE application layer protocol and published synchronously according to a periodic publishing rate regardless of a value change of a member in the data set. This method was previously suggested in [14] for wide-area networks and in this paper it is applied to process-level substation networks. Thereby, the probability of frame losses is reduced, since the spontaneously published GOOSE messages for status changes is disabled. Spontaneously published GOOSE message due to a status change usually coincides with many other status changes within the substation network and thereby a burst of GOOSE messages is sent, which increases the risk of network congestions. Furthermore, the GOOSE header comprises the UTC timestamp data attribute, mentioned in Tab. II. This UTC timestamp corresponds to the time validity of the encoded data, e.g. the point in time when the phasor estimation of the current and voltage signal has been performed. The remaining header fields of the GOOSE protocol are compared with the header fields of the SV protcol, as shown in Tab. IV. The size of these fields have been determined based on the ASN.1 Basic Encoding Rules (BER) and under the consideration of fixed-length GOOSE message encoding, as specified in IEC 61850-8-1 [16]. It should be mentioned that three octets
TABLE III

DATA SETS FOR PHASOR-BASED, TIME-DOMAIN BASED AND TRAVELLING-WAVE BASED TRANSMISSION LINE PROTECTION

Phasor-based transmission line protection PhsProtLine

\begin{tabular}{|c|c|c|c|}
\hline Data attribute & Size & Nr. & Total \\
\hline \multicolumn{4}{|l|}{ Base data set for all transmission lines } \\
\hline HARMXU.A.phsX.cVal.mag.i & 32 bit & $\mathrm{x} 4$ & 128 bit \\
\hline HARMXU.A.phsX.cVal.ang.i & 32 bit & $\mathrm{x} 4$ & 128 bit \\
\hline HARMXU.A.phsX.cVal.q & 32 bit & $\mathrm{x} 4$ & 128 bit \\
\hline HARMXU.PhV.phsX.cVal.mag.i & 32 bit & $\mathrm{x} 4$ & 128 bit \\
\hline HARMXU.PhV.phsX.cVal.ang.i & 32 bit & $\mathrm{x} 4$ & 128 bit \\
\hline HARMXU.PhV.phsX.cVal.q & 32 bit & $\mathrm{x} 4$ & 128 bit \\
\hline WSRMXU.StrCurVar.phsX & 1 bit & x3 & 3 bit \\
\hline WSRMXU.StrCurVar.q & 32 bit & $\mathrm{x} 1$ & 32 bit \\
\hline WSRMXU.StrMhoSup.phsX & 1 bit & $\mathrm{x} 3$ & 3 bit \\
\hline \multirow{2}{*}{ WSRMXU.StrMhoSup.q } & 32 bit & $\mathrm{x} 1$ & 32 bit \\
\hline & & & 838 bit \\
\hline \multicolumn{4}{|c|}{ Additional data for long transmission lines } \\
\hline HARMXU.A.phsX.cVal.mag.i & 32 bit & x3 & 96 bit \\
\hline HARMXU.A.phsX.cVal.ang.i & 32 bit & x3 & 96 bit \\
\hline \multirow[t]{2}{*}{ HARMXU.A.phsX.cVal.q } & 32 bit & x3 & 96 bit \\
\hline & & add. & 288 bit \\
\hline \multicolumn{4}{|c|}{ Additional data for transmission lines with in-zone transformers } \\
\hline HARMXU.HA.phsXHar.cVal.mag.i[] & 96 bit & $\mathrm{x} 4$ & 384 bit \\
\hline HARMXU.HA.phsXHar.cVal.ang.i[] & 96 bit & $\mathrm{x} 4$ & 384 bit \\
\hline HARMXU.HA.phsXHar.cVal.q & 32 bit & $\mathrm{x} 4$ & 128 bit \\
\hline TDRMXU.A.phsX.mag.i & 32 bit & x3 & 96 bit \\
\hline TDRMXU.A.phsX.q & 32 bit & $\mathrm{x} 3$ & 96 bit \\
\hline WSRMXU.StrWav.phsX & 1 bit & $\mathrm{x} 3$ & 3 bit \\
\hline \multirow[t]{2}{*}{ WSRMXU.StrWav.q } & 32 bit & $\mathrm{x} 1$ & 32 bit \\
\hline & & add. & 1123 bit \\
\hline \multicolumn{4}{|c|}{ Time-domain based transmission line protection } \\
\hline Data attribute & Size & Nr. & Total \\
\hline \multicolumn{4}{|l|}{ Data set TdProtLine } \\
\hline TDRMXU.TdDirFwdX.mag.i & 32 bit & $\mathrm{x} 3$ & 96 bit \\
\hline TDRMXU.TdDirFwdX.q & 32 bit & $\mathrm{x} 3$ & 96 bit \\
\hline TDRMXU.TdDirRevX.mag.i & 32 bit & x3 & 96 bit \\
\hline TDRMXU.TdDirRevX.q & 32 bit & $\mathrm{x} 3$ & 96 bit \\
\hline TDRMXU.TdDirOpX.mag.i & 32 bit & x3 & 96 bit \\
\hline TDRMXU.TdDirOpX.q & 32 bit & x3 & 96 bit \\
\hline TDRMXU.TdDisValX.mag.i & 32 bit & $\mathrm{x} 6$ & 192 bit \\
\hline \multirow[t]{2}{*}{ TDRMXU.TdDisValX.q } & 32 bit & $\mathrm{x} 6$ & 192 bit \\
\hline & & & 960 bit \\
\hline
\end{tabular}

Travelling-wave based transmission line protection

\begin{tabular}{|l|r|r|r|r|}
\hline Data attribute & Size & Nr. & Total \\
\hline Data set TwProtLine & 32 bit & x3 & 96 bit \\
\hline TWRMXU.TwDirValX.mag.i & 32 bit & x3 & 96 bis \\
\hline TWRMXU.TwDirValX.q & 32 bit & x3 & 96 bit \\
\hline TWRMXU.TwDifValX.mag.i & 32 bit & x3 & 96 bit \\
\hline TWRMXU.TwDifValX.q & & & $\mathbf{3 8 4}$ bit \\
\hline
\end{tabular}

have been considered for the BER length field of goosePdu, allData, savPdu and seqASDU. Moreover, the length of the value fields of gocbRef and datSet has been considered to be 10 and of the goID to be 2 bytes. Finally, the GOOSE and SV headers together with their respective datasets are enclosed in an Ethernet frame. The overhead of Ethernet has been estimated to be 42 bytes, which includes the 4 bytes 802.1Q tag and the 12 bytes interpacket gap.

\section{AnAlysis of the COMmunication LOAD OF THE PROCESS-LEVEL NETWORK}

This section analyses analytically the impact on the communication load of the process-level substation network as 
TABLE IV

GOOSE AND SV OVERHEAD CONSIDERING BER ENCODING

\begin{tabular}{|c|c|c|c|}
\hline GOOSE header field & Size & SV header field & Size \\
\hline goosePdu & 4 bytes & savPdu & 4 bytes \\
\hline gocbRef & 12 bytes & noASDU & 3 bytes \\
\hline timeAllowedToLive & 7 bytes & seqASDU & 4 bytes \\
\hline datSet & 12 bytes & ASDU & 2 bytes \\
\hline goID & 4 bytes & svID & 4 bytes \\
\hline $\mathrm{t}$ & 10 bytes & smpCnt & 4 bytes \\
\hline stNum & 7 bytes & confRev & 6 bytes \\
\hline sqNum & 7 bytes & smpSynch & 3 bytes \\
\hline simulation & 3 byte & - & \\
\hline confRev & 7 bytes & - & - \\
\hline ndsCom & 3 byte & - & - \\
\hline numDatSetEntries & 7 bytes & - & \\
\hline allData & 4 bytes & seqData & 2 bytes \\
\hline Total & 87 bytes & Total: & 32 bytes \\
\hline
\end{tabular}

well as describes the different sampling and publishing rate variants of SV. Subsequently, these variants are considered as a reference in order to compare the DSPU-based and the MUbased process-level network concepts.

\section{A. Sampled Value Reference Cases}

The data set for the Sampled Values is specified in IEC 61869-9 [11] and comprises 4 current and 4 voltage samples per ASDU, which are mapped to the value field of seqData. Furthermore the standard IEC 61869-9 defines different variants of digital sampling rates and publishing rates according to the notation $\mathrm{F} f \mathrm{~S} s \mathrm{I} i \mathrm{U} u$, where

$f$ digital sampling rate.

$s$ number of ASDUs in a sampled value message.

$i$ number of current samples in each ASDU.

$u$ number of voltage sampels in each ASDU.

This paper considers the recommended variants by the standard corresponding to F4800S2I4U4, F14400S6I4U4 and F96000S1I4U4 that are used as reference cases for the phasorbased, the time-domain based and the travelling-wave based protection, respectively. The size of the SV data set equals 64 bytes. It should be noticed that the BER encoding rules are applied for the GOOSE data sets, specified in Tab. III, but not for the the SV data set.

\section{B. Results of the Network Bandwidth Estimation}

Based on the reference cases in Sec. IV-A and the derived data sets in Sec. III-B, the generated communication load by the DSPU is compared with the communication load produced by the MU. This comparison is made for three different types of protection functions, namely phasor-based protection, additional time-domain based protection and additional travellingwave based protection, as shown respectively in Fig. 2, in Fig. 3 and in Fig. 4. Moreover, the three different data sets for the phasor-based protection, shown in Tab. III-B, are considered, reflecting the information requirements for a base and long transmission line as well as for a transmission line with an in-zone transformer.

In Fig. 2 the required network bandwidth is shown with respect to a publishing rate from $1 \mathrm{~ms}$ to $5 \mathrm{~ms}$ of the DSPU. It can be

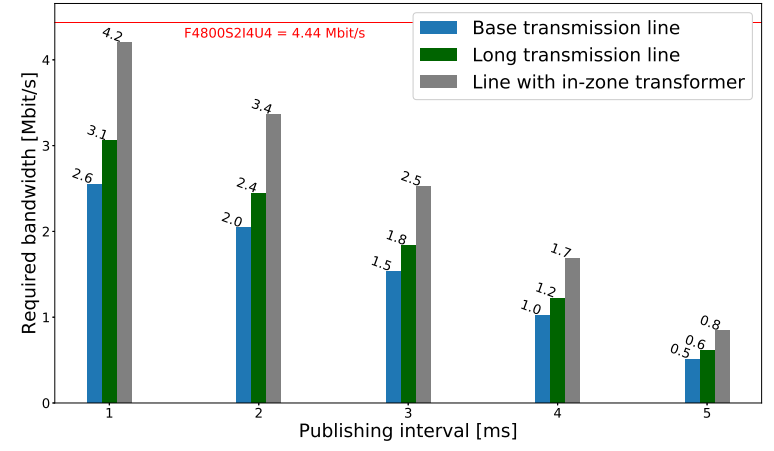

Fig. 2. Communication load for phasor-based protection

seen that the communication load is reduced for all three types of transmission lines as compared to the $4.8 \mathrm{kHz} \mathrm{SV}$-stream, i.e. F4800S2I4U4. Nonetheless, the margin is not significant for the phasor-based protection functions.

A similar result is obtained considering an additional data load due to the time-domain protection data set, as shown in Fig. 3 . In this case, the comparison is made with the SV-stream of $14.4 \mathrm{kHz}$, i.e. F14400S6I4U4, due to the increased signal spectrum requirements of time-domain protection applications [6]. Fig. 3 shows an increasing reduction of the required network bandwidth with respect to a decreasing publishing rate.

The required network bandwidth for an additional data load due to the travelling-wave protection data set is shown in Fig. 4. It should be noted that the publishing rate of the DSPU is plotted from $100 \mu$ s to $1 \mathrm{~ms}$ due to the speed requirements of travelling-wave based protection. Fig. 4 indicates a significant reduction of the required network bandwidth in comparison to the MU-based F96000S1I4U4 reference case. Thus the advantage of the DSPU concept becomes evident.

Lastly, the impact on the required network bandwidth due to an increasing number of DSPUs and MUs, respectively, has been analysed in Fig. 5. In this case a publishing rate of $1 \mathrm{~ms}$ is assumed for the phasor-based and time-domain based protection data sets and a publishing rate of $100 \mu$ s for the travelling-wave based protection data set. If only phasorbased protection functions are deployed, the DSPU approach results only in a marginal reduction of the required network bandwidth in comparison to the MU approach considering 1 Gigabit/s process-level substation network. Nevertheless, the DSPU concept reduces the network bandwidth clearly for the high sampling rate applications, such as time-domain based and travelling-wave based protection. Especially for substation automation systems with a high number of travelling-wave based protection functions the maximum number of MUs, publishing SV according to F96000S1I4U4, would be limited to below nine and in practice an even lower number would be obtained due to other traffic on the network.

\section{Discussion}

The results show that the communication load of the process-level network can be significantly reduced by applying 


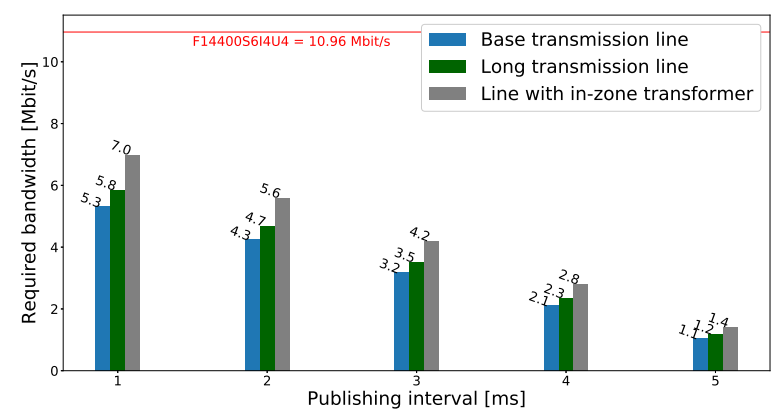

Fig. 3. Communication load for time-domain based protection

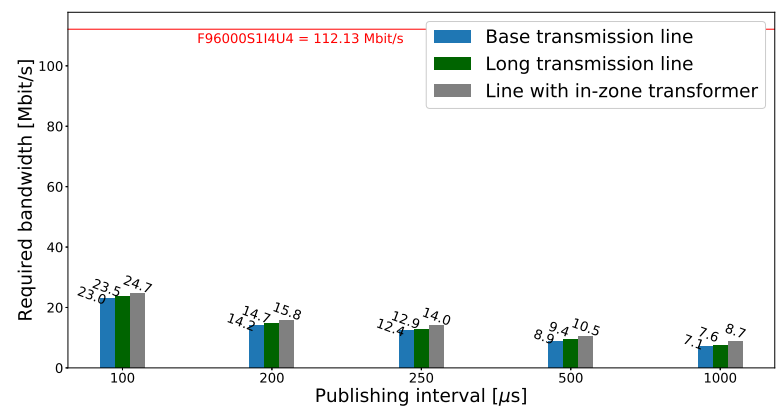

Fig. 4. Communication load for travelling-wave based protection

the DSPU concept in comparison to the MU approach. This becomes evident for high sampling rate applications, since the publishing rate of the DSPU is independent of the sampling rate of its input signals. The data load of the data set due to travelling-wave based protection has been compared to the variant F96000S1I4U4 corresponding to a sampling rate of $96 \mathrm{kHz}$. Nonetheless, some travelling-wave based protection applications require sampling rates up to $1 \mathrm{MHz}$, which makes the direct publishing of raw samples very bandwidth demanding. Thus the DSPU concept is very applicable for these high sampling rate protection applications.

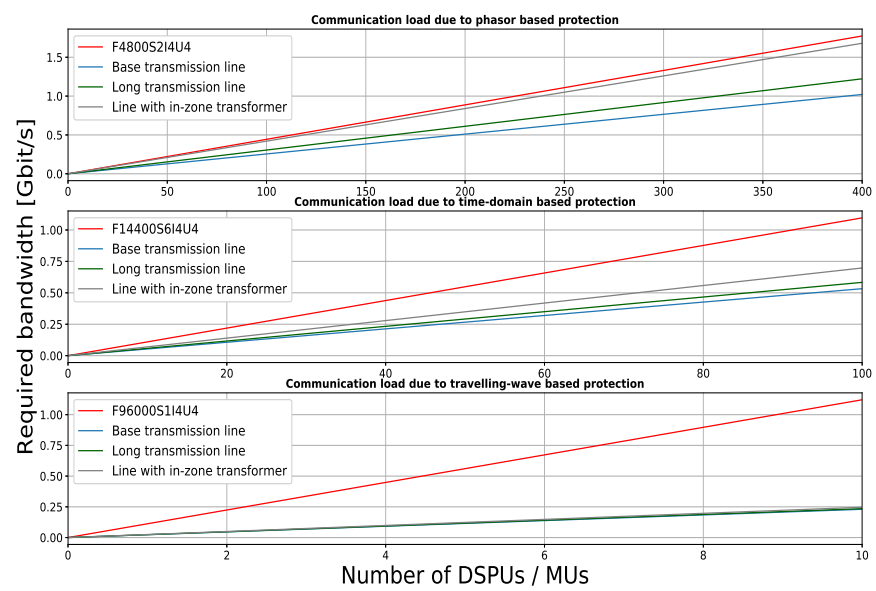

Fig. 5. Required bandwidth considering a DSPU publishing rate of $1 \mathrm{~ms}$

\section{CONCLUSION}

This paper has proposed data models for the DSPU in terms of IEC 61850 logical nodes and data sets, which are mapped to the GOOSE application layer protocol. Additionally, the impact on the communication load of the process-level network has been analysed for different sampling rate protection applications. It has been shown that the DSPU concept can significantly reduce the required network bandwidth for protection applications that require high sampling rates, such as travelling-wave based protection.

\section{REFERENCES}

[1] D. McGinn, M. Adamiak, M. Goraj, and J. Cardenas, "Reducing conventional copper signaling in high voltage substations with IEC 61850 process bus system," in 2009 IEEE Bucharest PowerTech, June 2009, pp. $1-8$.

[2] IEC 61850-5:2013 Communication networks and systems for power utility automation - Part 5: Communication requirements for functions and device models, TC 57 Std., 2013.

[3] D. M. E. Ingram, D. A. Campbell, and P. Schaub, "Use of IEEE 15882008 for a sampled value process bus in transmission substations," in 2011 IEEE International Instrumentation and Measurement Technology Conference, May 2011, pp. 1-6.

[4] A. d. Santos, B. Soares, C. Fan, M. Kuipers, S. Sabino, A. M. R. C Grilo, P. R. B. A. Pereira, M. S. Nunes, and A. Casaca, "Characterization of substation process bus network delays," IEEE Transactions on Industrial Informatics, vol. 14, no. 5, pp. 2085-2094, May 2018.

[5] S. Brahma, "Advancements in centralized protection and control within a substation," IEEE Transactions on Power Delivery, vol. 31, no. 4, pp. 1945-1952, Aug. 2016.

[6] E. O. Schweitzer, B. Kasztenny, and M. V. Mynam, "Performance of time-domain line protection elements on real-world faults," in 2016 69th Annual Conference for Protective Relay Engineers (CPRE), April 2016, pp. 1-17.

[7] N. Jiang, W. Bin, and D. Xinzhou, "Travelling wave sampling data sharing solution in distribution substation based on IEC61850," in 12th IET International Conference on Developments in Power System Protection (DPSP 2014), March 2014, pp. 1-5.

[8] IEC 61850-90-4:2013 Communication networks and systems for power utility automation - Part 90-4: Network engineering guidelines, TC 57 Std., 2013.

[9] "IEEE recommended practice for signal treatment applied to smart transducers," IEEE Std 21451-001-2017, pp. 1-109, Dec 2017.

[10] F. Hohn, J. Wang, and L. Nordström, "Distributed signal processing units for centralized substation protection and control," in 14th IET International Conference on Developments in Power System Protection (DPSP 2018), March 2018, in press.

[11] IEC 61869-9:2016 Instrument transformers - Part 9: Digital interface for instrument transformers, TC 38 Std., 2016.

[12] IEC 61850-7-4:2013 Communication networks and systems for power utility automation - Part 7-4: Basic communication structure Compatible logical node classes and data object classes, TC 57 Std., 2010.

[13] IEC 61850-7-3:2013 Communication networks and systems for power utility automation - Part 7-3: Basic communication structure Common data classes, TC 57 Std., 2013.

[14] J. M. Herrera, M. S. Mingarro, S. L. Barba, D. Dolezilek, F. Calero, A. Kalra, and B. Waldron, "Case study of time-domain automation and communications: Field-proven benefits to automation, control, monitoring, and special protection schemes," in 2017 Saudi Arabia Smart Grid (SASG), Dec 2017, pp. 1-8.

[15] C. R. Ozansoy, A. Zayegh, and A. Kalam, "The real-time publisher/subscriber communication model for distributed substation systems," IEEE Transactions on Power Delivery, vol. 22, no. 3, pp. 14111423, July 2007.

[16] IEC 61850-8-1:2011 Communication networks and systems for power utility automation - Part 8-1: Specific communication service mapping (SCSM) Mappings to MMS (ISO 9506-1 and ISO 9506-2) and to ISO/IEC 8802-3, TC 57 Std., 2011. 